\title{
Immune-Related DNA Methylation Data-Based Molecular Classification Associated with the Prognosis of Patients with Hepatocellular Carcinoma
}

\section{Xiong-Wen Wang ( $\sim$ awen681029@163.com )}

Guangzhou University of Traditional Chinese Medicine First Affiliated Hospital https://orcid.org/00000002-5428-7110

\section{Qian Yan}

Guangzhou University of Chinese Medicine

\section{Bao-Qian Ye}

Guangzhou University of Chinese Medicine

\section{Bo-Qing Wang}

Guangzhou University of Chinese Medicine

\section{Wen-Jiang Zheng}

Guangzhou University of Chinese Medicine

\section{Research article}

Keywords: hepatocellular carcinoma, DNA methylation, molecular subtype, immune-related genes; prognosis model.

Posted Date: May 18th, 2021

DOI: https://doi.org/10.21203/rs.3.rs-531042/v1

License: (9) This work is licensed under a Creative Commons Attribution 4.0 International License. Read Full License 


\section{Abstract}

Background: The combination of epigenetic drugs and immunotherapy should be able to develop an optimal treatment plan for hepatocellular carcinoma ( $\mathrm{HCC})$, yet its mechanism is still in the preliminary exploration stage. The purpose of this study is to analyze the DNA methylation and gene expression profiles of immune-related $\mathrm{CpG}$ sites to identify the molecular subtypes and $\mathrm{CpG}$ sites related to the prognosis of HCC.

Methods: In this study, the DNA methylation and gene expression datasets were downloaded from The Cancer Genome Atlas database, together with immune-related genes downloaded from the immunology database and analysis portal database to explore the prognostic molecular subtypes of HCC. Univariate and multivariate survival analysis was used for selecting the significant methylation sites, and the consensus clustering was performed to find the best molecular subtype associated with the survival of HCC. Next, we used the least absolute shrinkage and selection operator (LASSO) algorithm to construct a prognostic-related model and performed internal verification. Finally, we explored the levels of 16 immune-related genes expression correlate with the infiltration levels of immune cells in HCC.

Results: By performing consistent clustering analysis on 830 immune-related $\mathrm{CpG}$ sites in 231 samples of a training set, we identified seven subgroups with significant differences in overall survival. Finally, 16 classifiers of immune-related $\mathrm{CpG}$ sites were constructed and used in the testing set to verify the prognosis of DNA methylation subgroups, and the results were consistent with the training set. Using the TIMER database, we analyzed 16 immune-related $\mathrm{CpG}$ sites expression with the abundance of six types of immune infiltrating cells and found that most are positively correlated with the level of infiltration of multiple immune cells in HCC. Conclusions: This study screened potential immune-related prognostic methylation sites and established a new prognosis model of HCC based on DNA methylation molecular subtype, which may help in the early diagnosis of HCC and developing more effective personalized treatments.

\section{Full Text}

This preprint is available for download as a PDF.

\section{Figures}




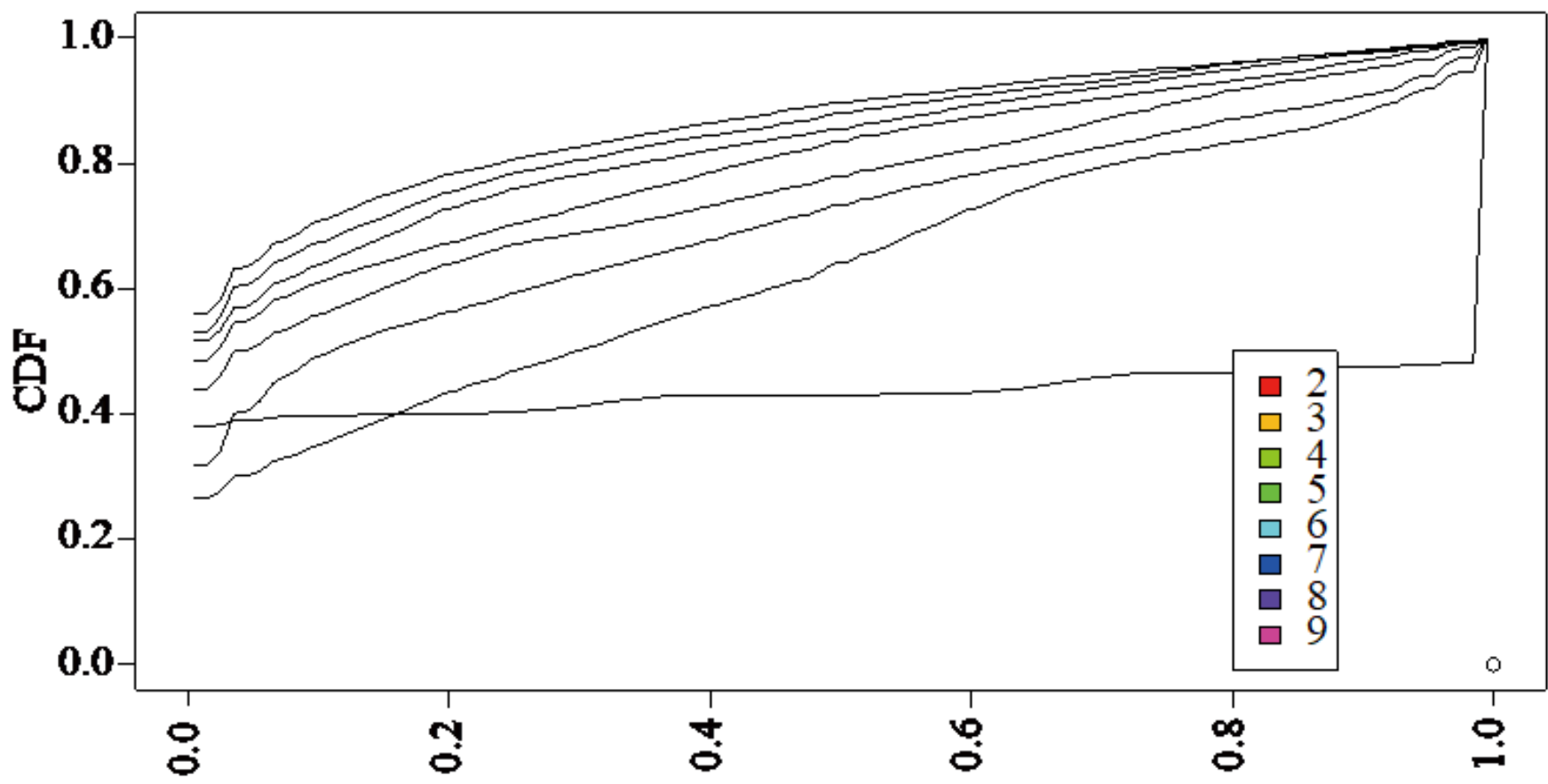

Consensus index

B

Delta area

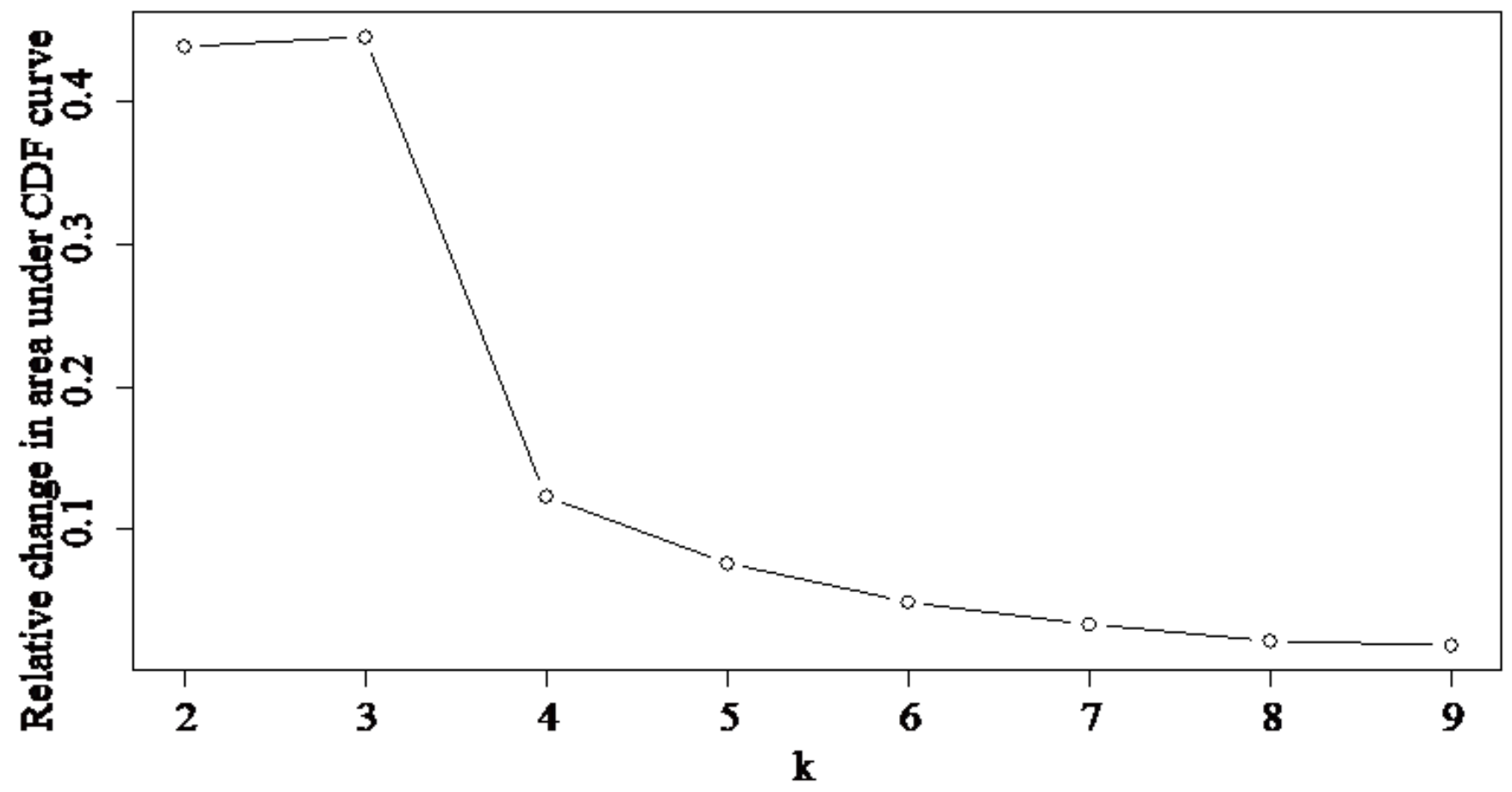

Figure 1

The consensus cluster analysis of various DNA methylation prognosis subgroups in hepatocellular carcinoma. (A) The consensus cumulative distribution function (CDF) curve among clusters for every category number k. (B) The Delta area curves for consensus clustering (y-axis represents the relative change in area under the CDF curve, and the abscissa means the category number $k$ ), which indicates the relative change in area under the CDF curve for each category number k compared to k - 1. 

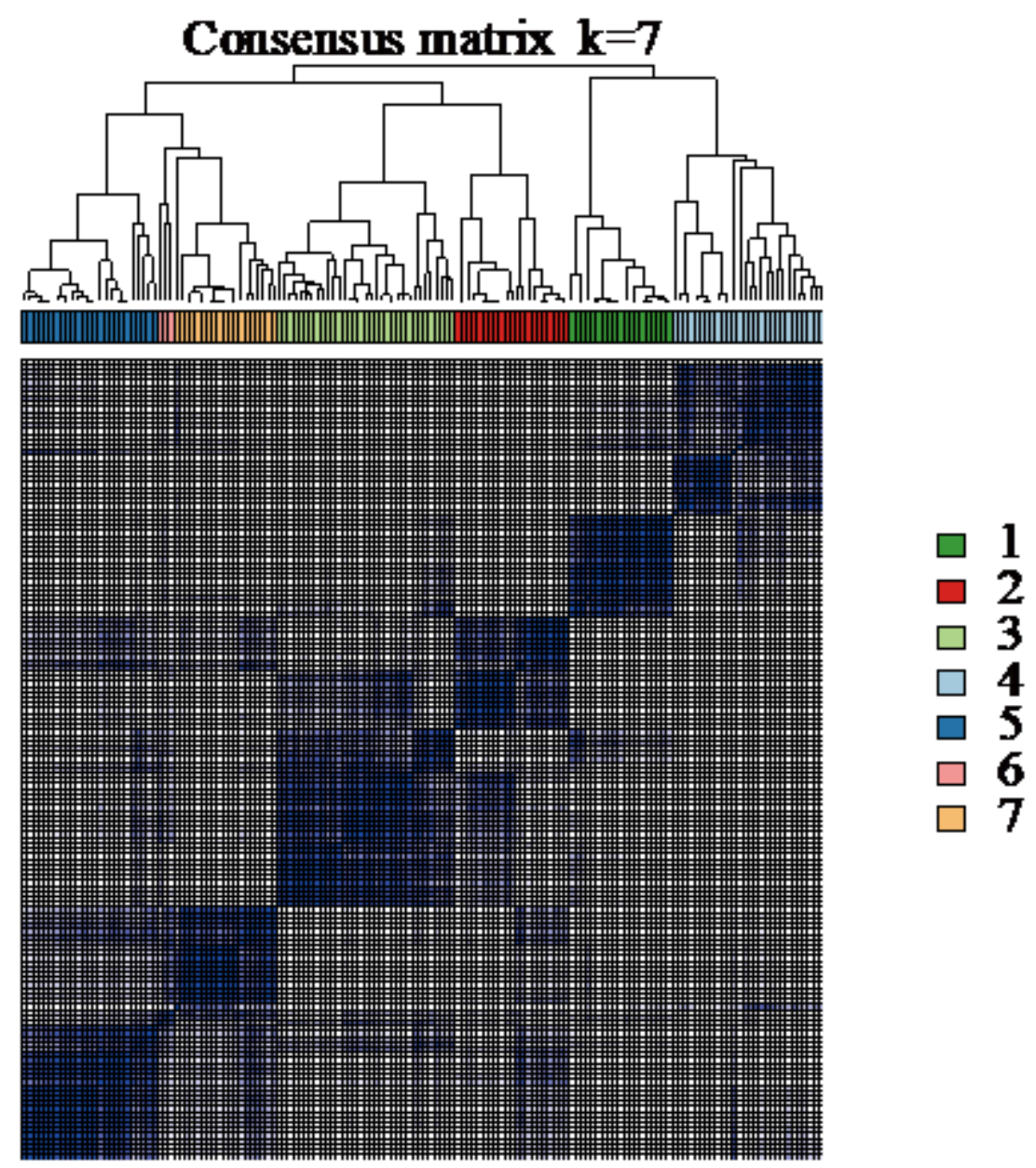

Figure 2

The color-coded heatmap of the consensus matrix for seven molecular subgroups classification obtained by applying the consensus cluster ( 1 to 7 in the legend represent Cluster 1 to 7 ); the color gradient indicates the consensus value from 0 to 1 ; white indicates 0 , and dark blue indicates 1 ). 


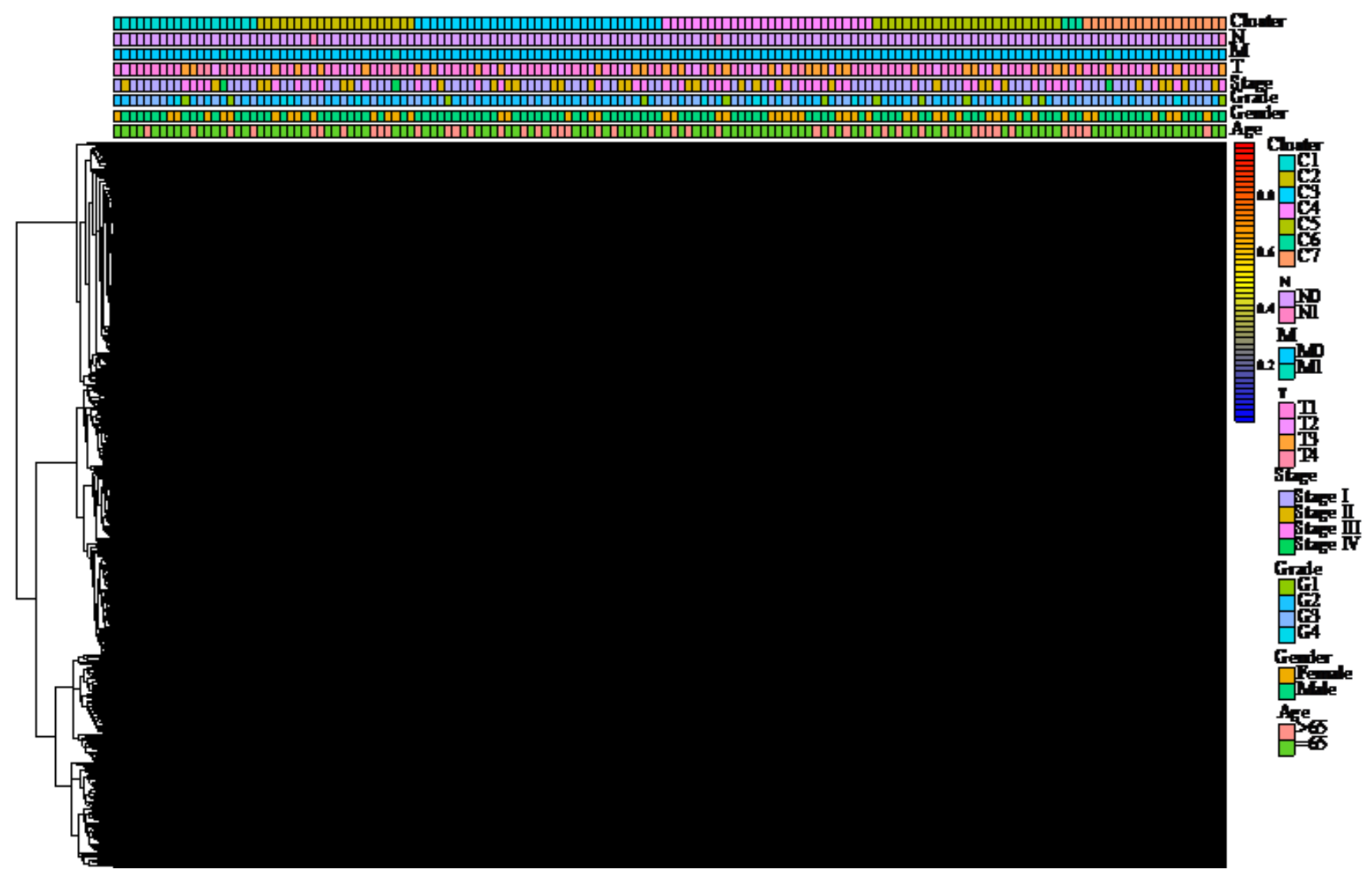

Figure 3

The heatmap of 840 methylation sites of the seven clusters (the heatmap combined DNA methylation classification with age, gender, TNM stage, and clinicopathological stage as the annotations). 
A

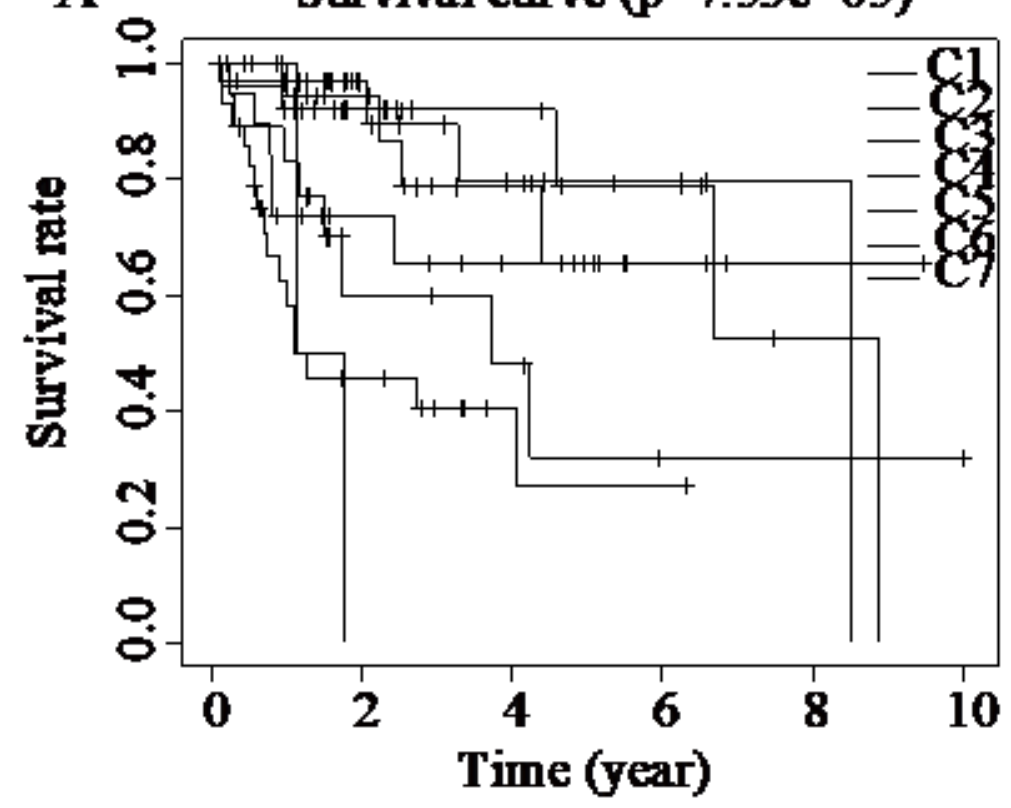

Survival curve $(p=7.53 \mathrm{e}-05)$

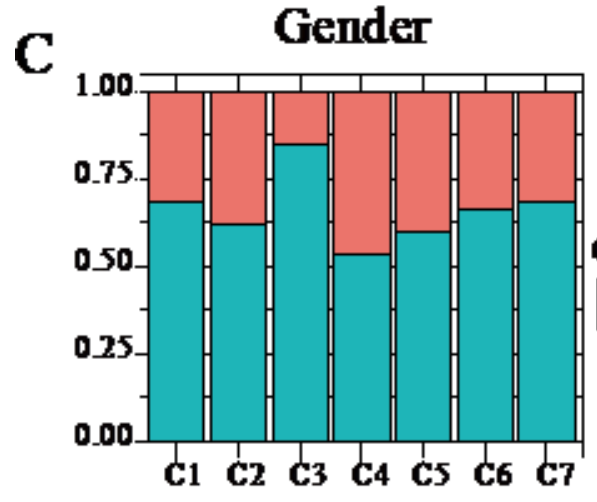

Cluster

F

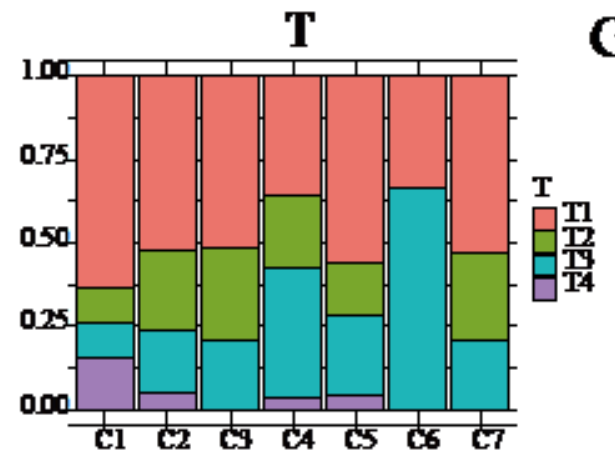

Cluster

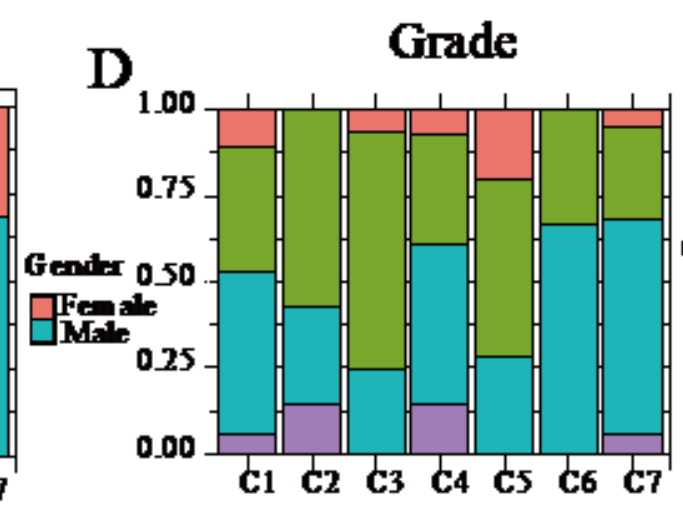

Cluster

G

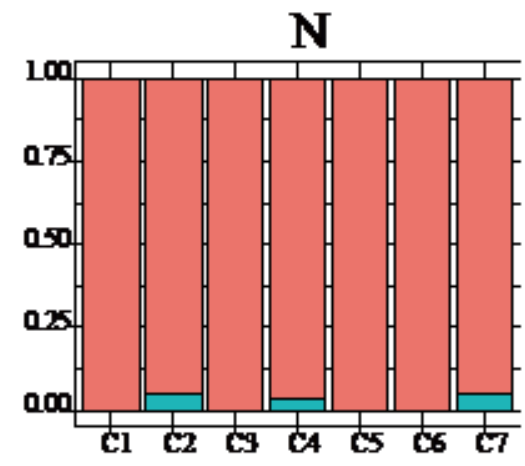

Cluster
B Age

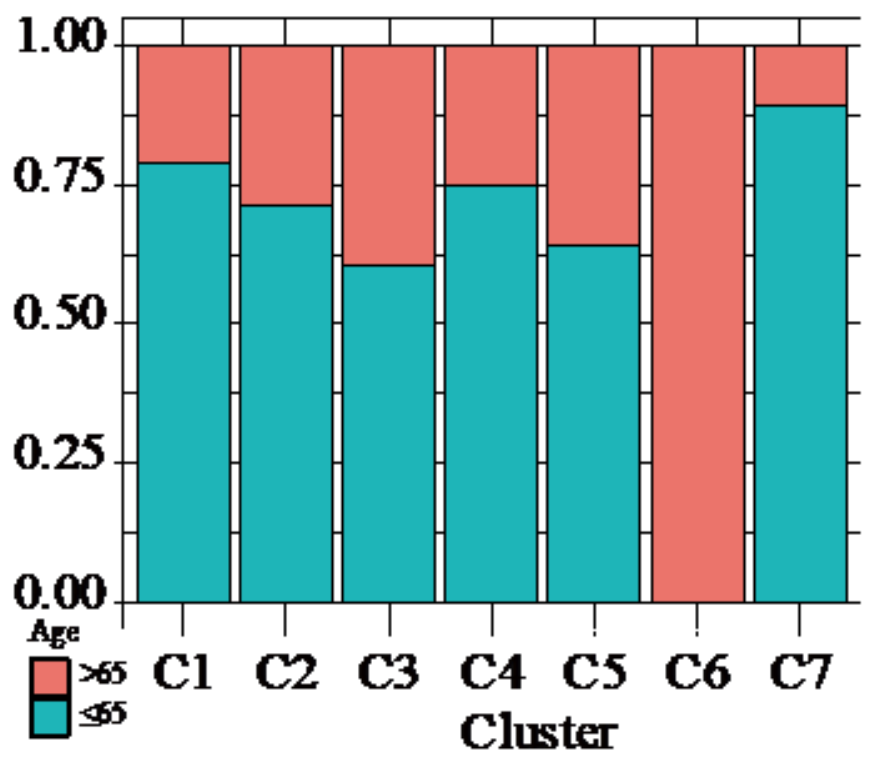

E
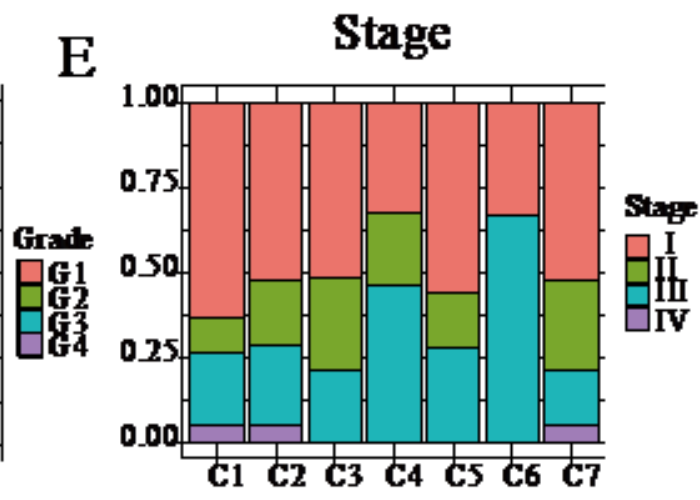

Cluster

H

M

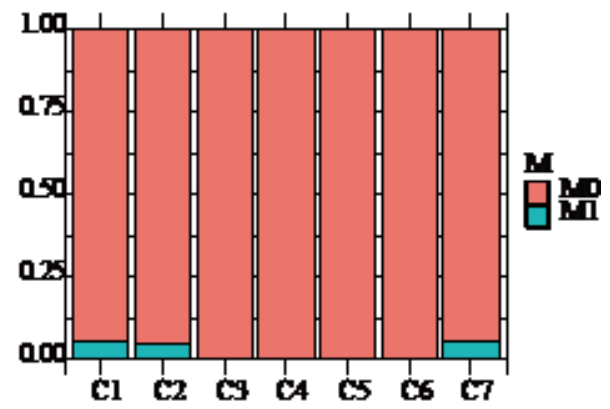

Cluster

Figure 4

The prognostic difference and the distribution of different clinical factors among seven clusters. (A) Prognosis difference among seven clusters. The horizontal axis represents the survival time (year), the vertical axis represents the survival rate, and the $p$-value represents the significance of the difference between the clusters. Distribution proportion of age (B), gender (C), grade (D), stage (E), T staging (F), N staging $(\mathrm{G})$, and $M$ staging $(\mathrm{H})$ among seven clusters. 


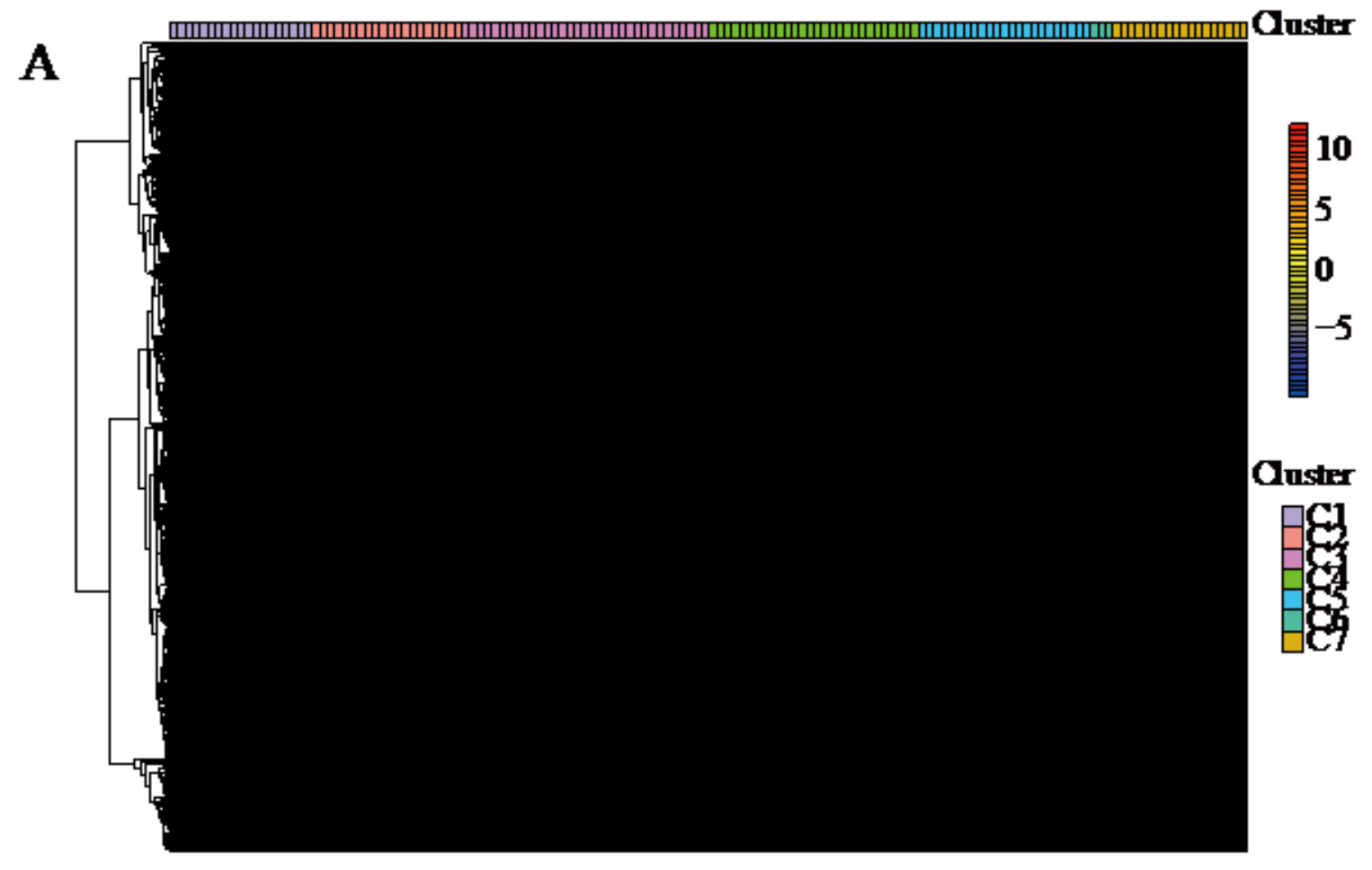

B

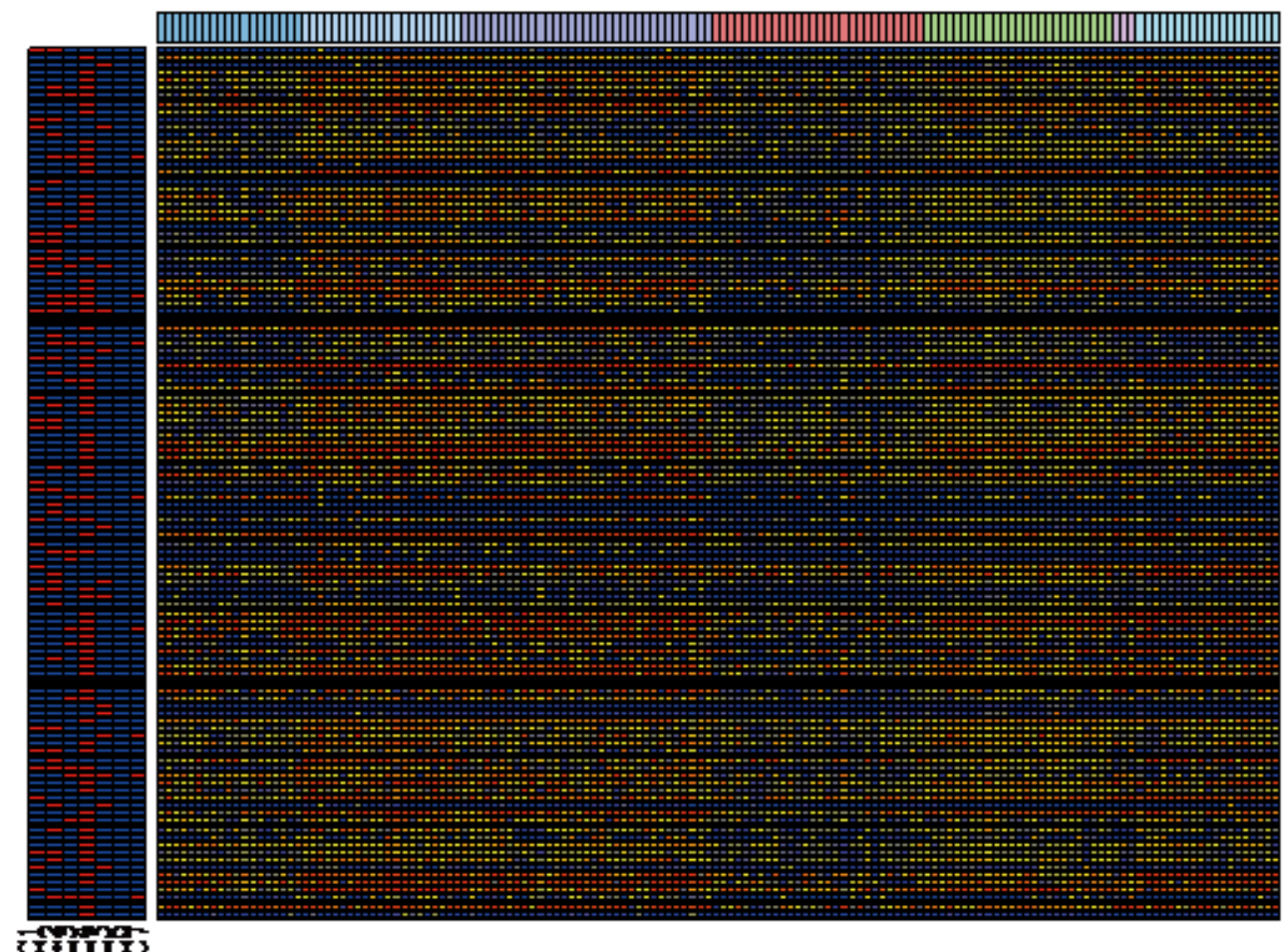

Ouster

Difference

$\square$ No

$\square$ Sig

Methylation

0.8

$8-6$

02

Cluster

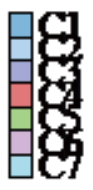

Figure 5

The heatmap of the gene expression of specific CpG sites and the methylation level of differential methylation sites among the seven clusters. (A) Heatmap of 513 gene expressions in seven DNA methylation clusters. (B) Heatmap of differential methylation levels of 238 specific CpG sites for each DNA methylation prognosis subtype. The red bars represent hypermethylated CpG sites or 
hypomethylated $\mathrm{CpG}$ sites, and the blue bars indicate there was no significance in the methylation level of $\mathrm{CpG}$ sites among all clusters.

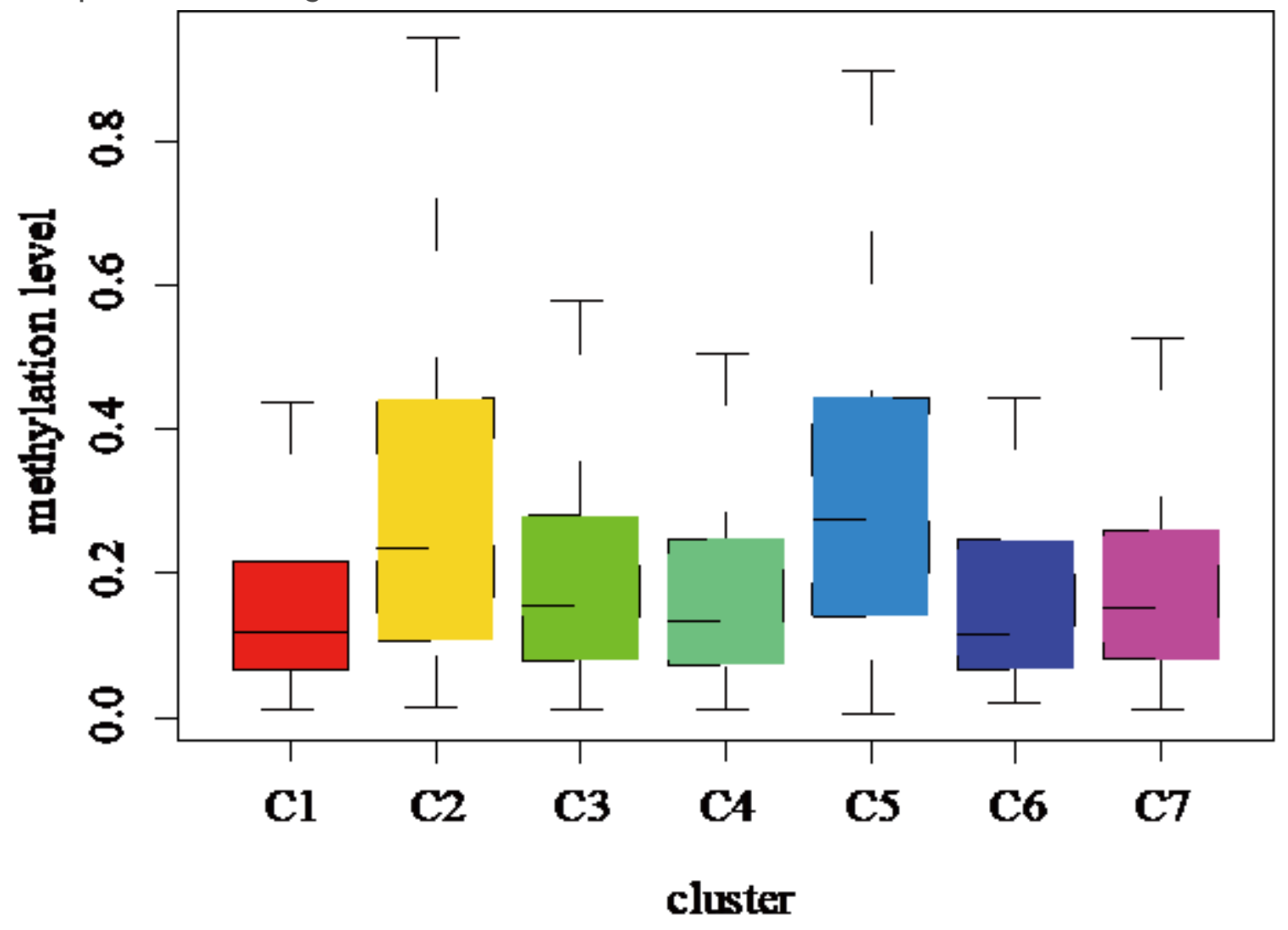

Figure 6

Box plot of the methylation level (z-score) of $\mathrm{CpG}$ sites in the seven clusters (compared to other clusters, cluster 5 has the highest methylation level). 


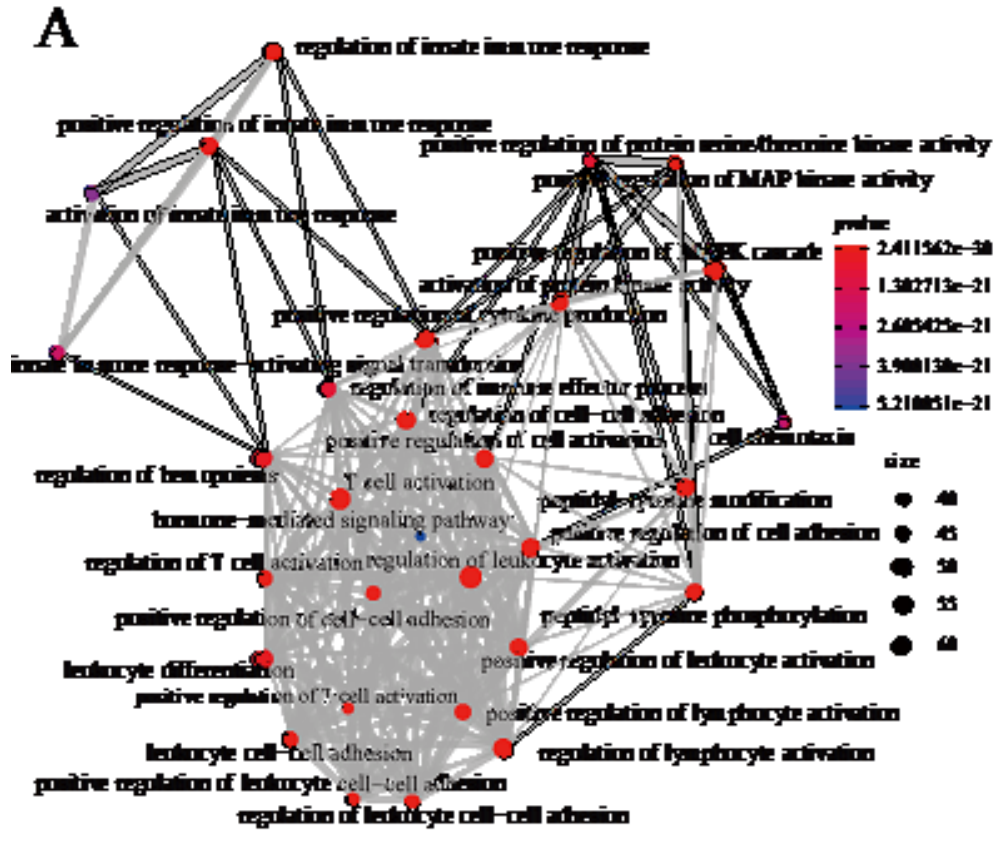

C

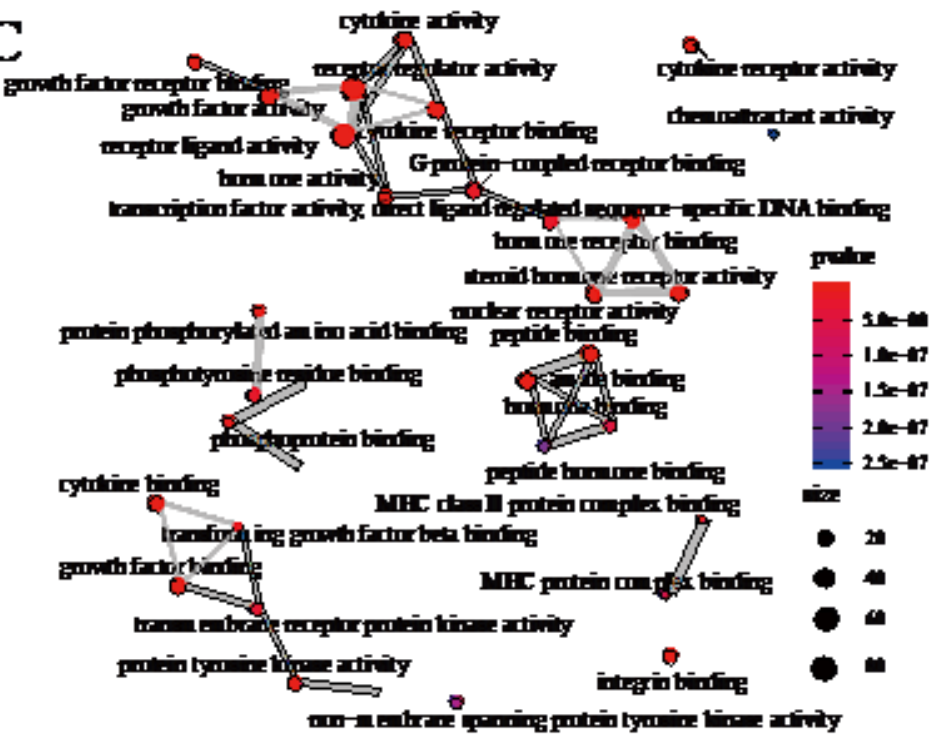

B
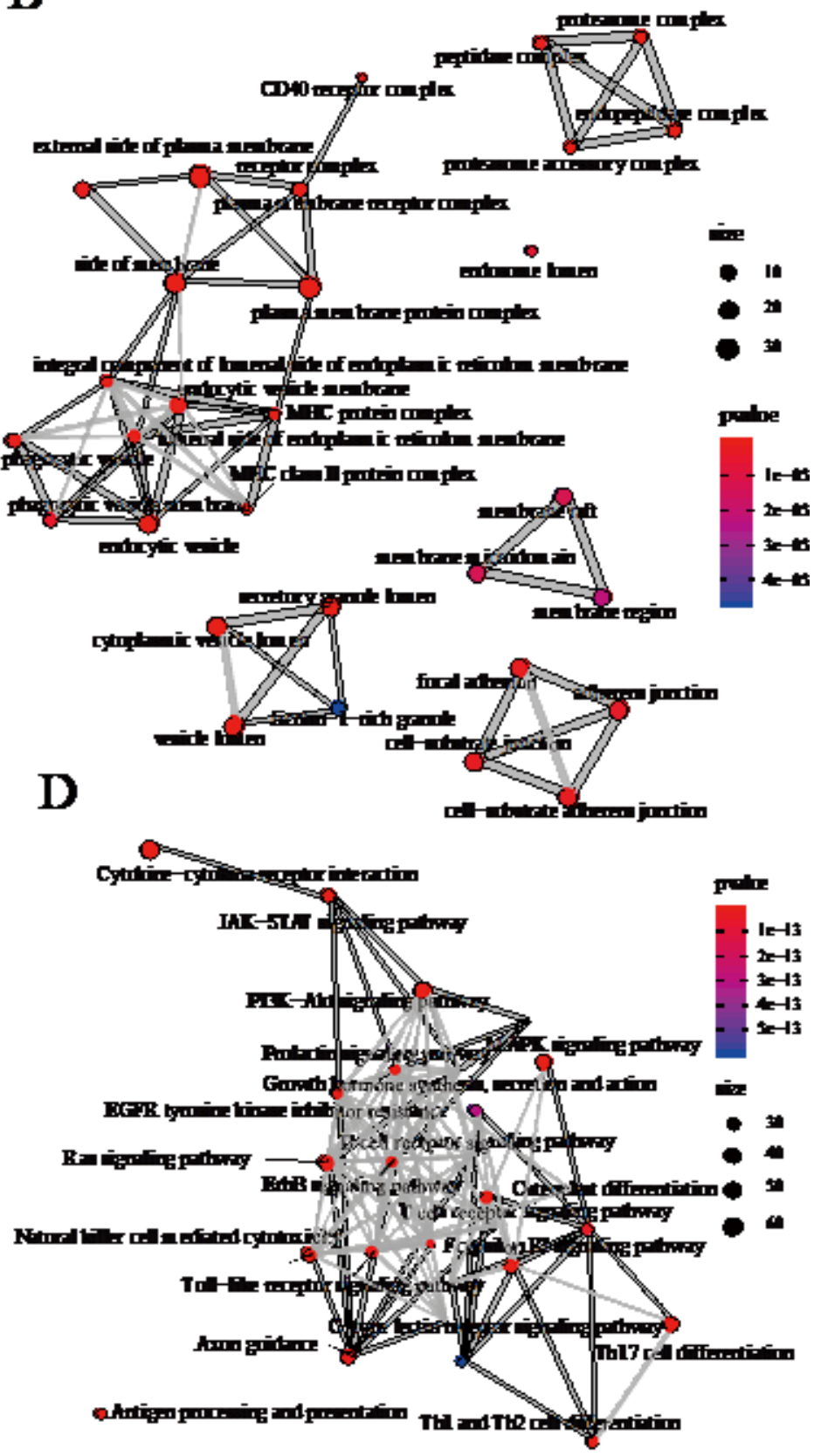

Figure 7

Gene ontology (GO) and Kyoto Encyclopedia of Genes and Genomes (KEGG) enrichment analysis of 840 annotated genes associated with prognosis methylation sites (the size of the dots is proportional to the number of genes enriched; the color of the dot is the degree of significance, the degree of significance gradually increases from red to blue, and the line indicates that there is a correlation between the two entries). (A) The first 30 items enriched by the biological process in $\mathrm{GO}$ analysis. (B) The first 30 items enriched by cellular component in $\mathrm{GO}$ analysis. (C) The first 30 items enriched in molecular function in GO analysis. (D) The first 20 items enriched in KEGG analysis. 

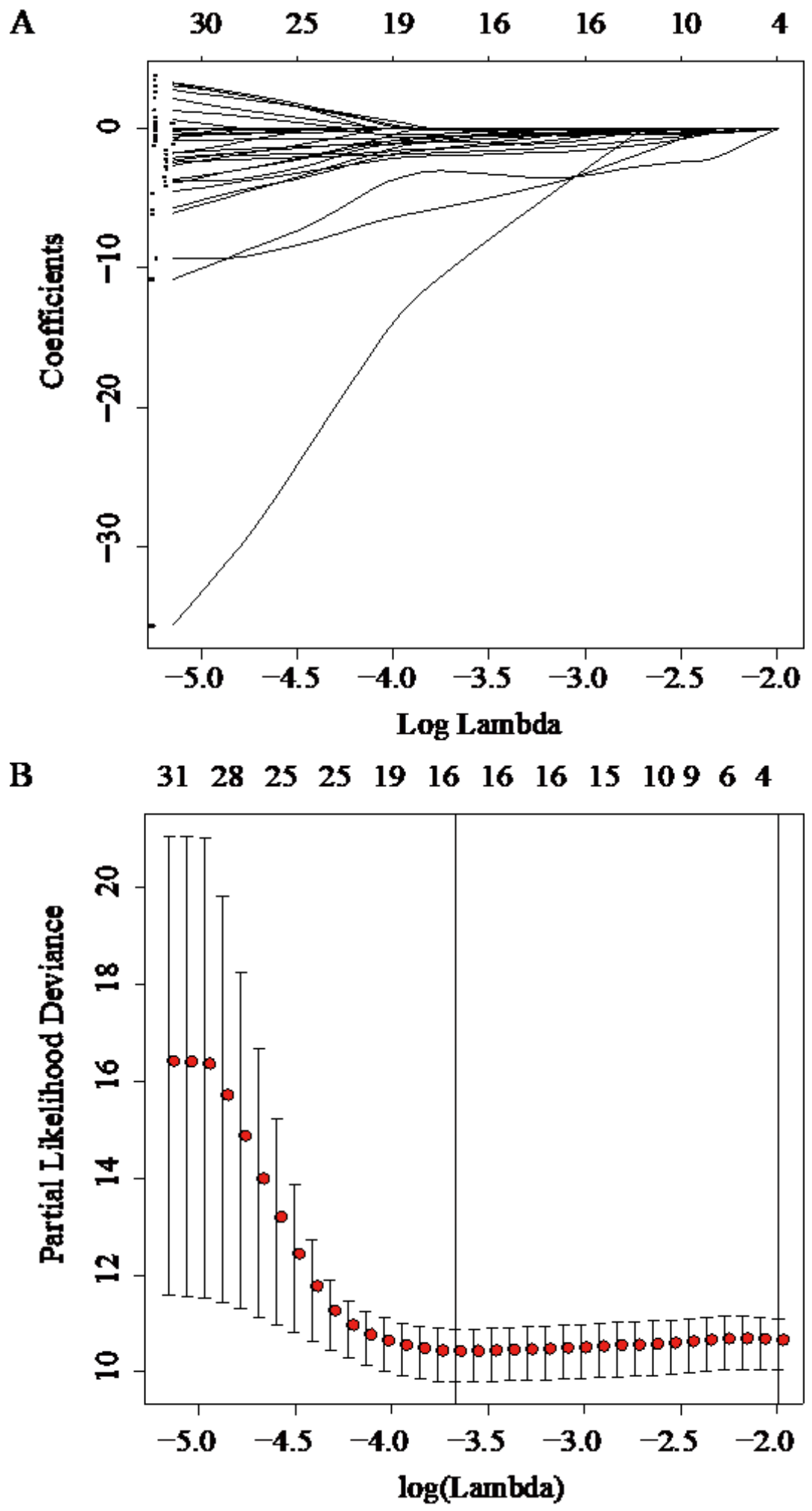

Figure 8

The least absolute shrinkage and selection operator regression constructs a prognostic model of DNA methylation sites associated with immune genes in hepatocellular carcinoma. (A) Change track of each independent variable. The horizontal axis represents the logarithmic value of the independent variable $\lambda$, and the vertical axis represents the coefficient of the independent variable. (B) The confidence interval of each $\lambda$; the $x$-axis represents the range of $\lambda$ values, and the $y$-axis represents the partial likelihood 
deviance; when the $y$-axis takes the minimum values, the $\lambda$ value and the number above the $x$-axis are the optimal numbers of prognostic models.

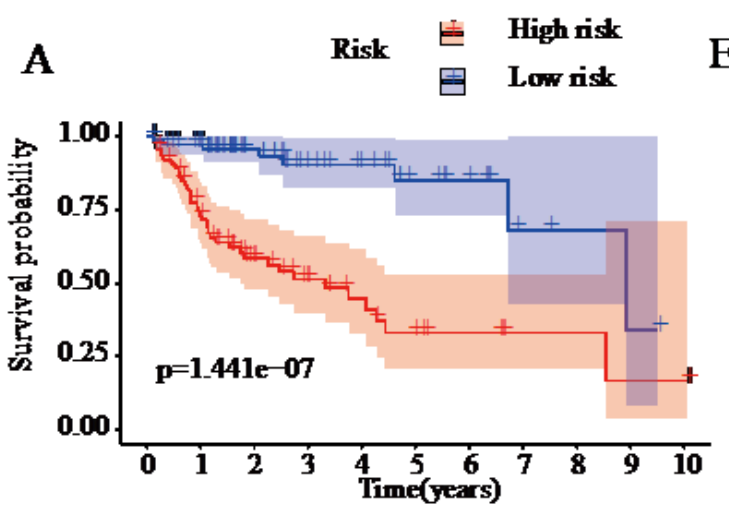

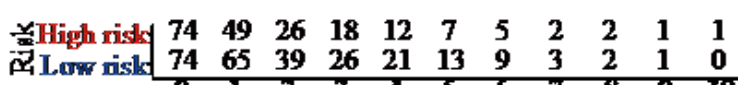

B
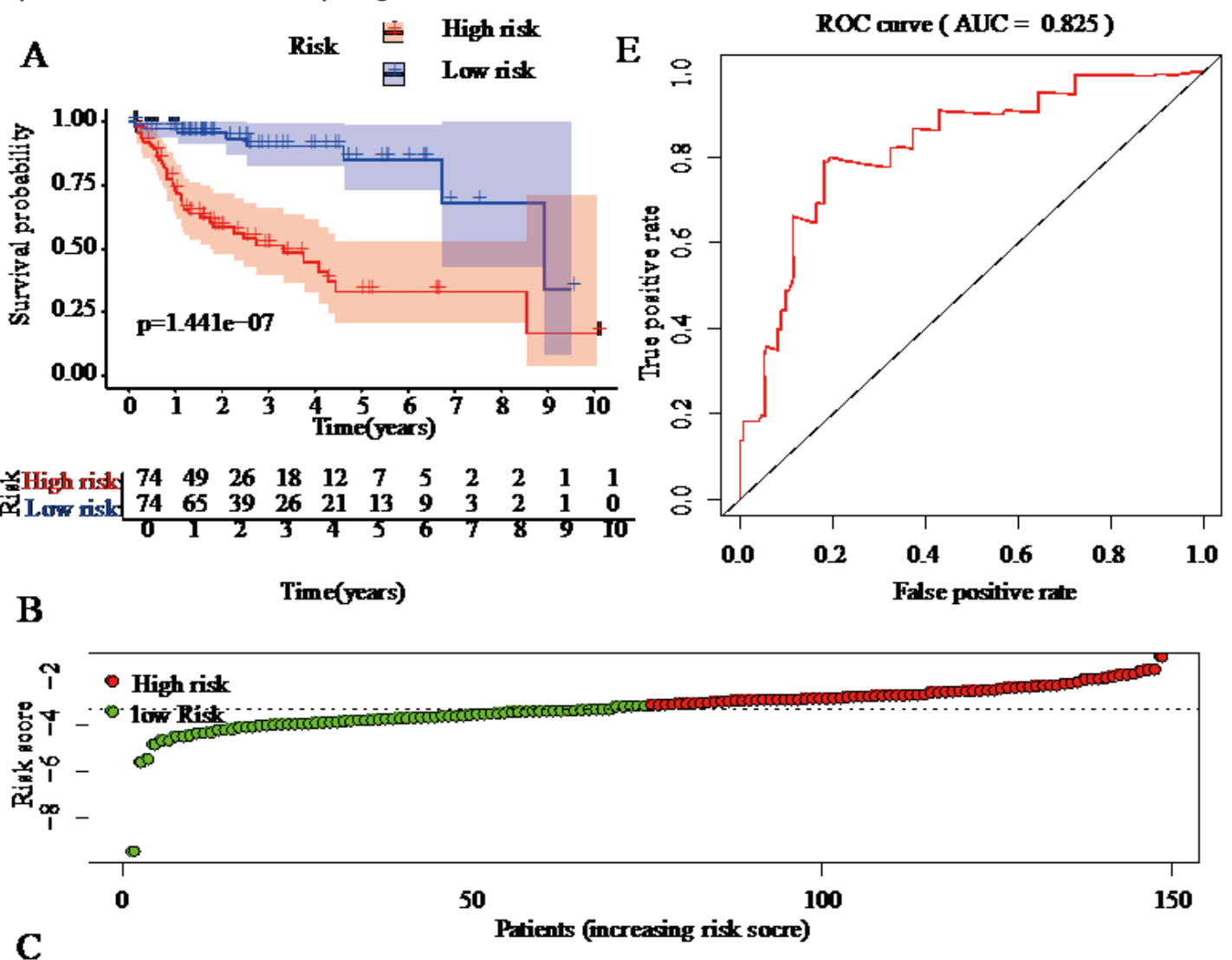

C

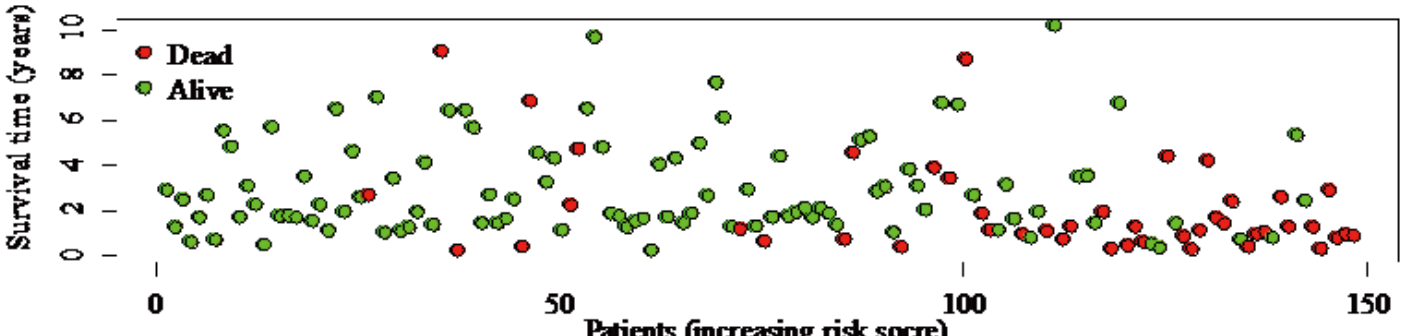

D

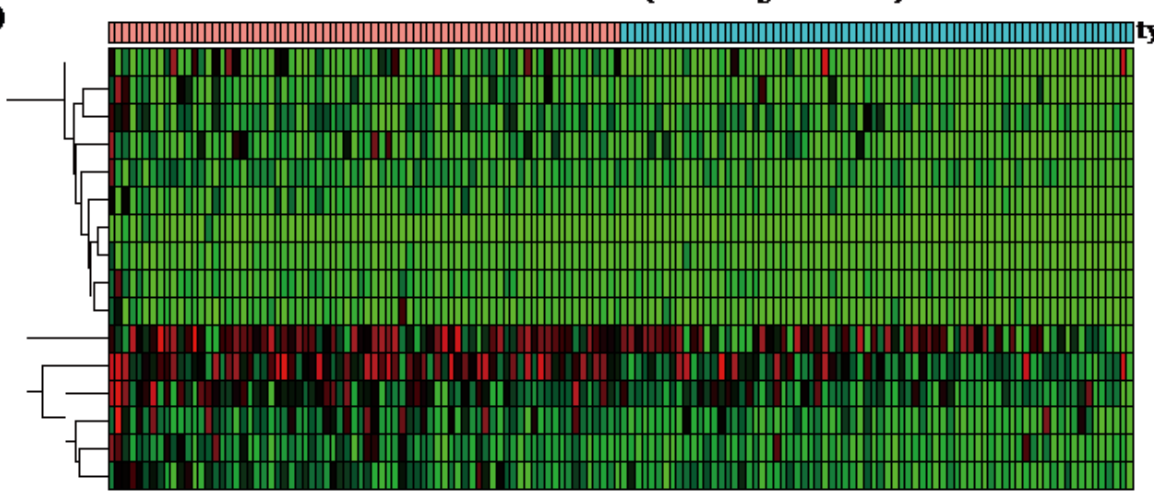

cg15140465

cg15929078

cg19476647 0.8

cg13615963

cg01914037

cg00536939

cg09959112

cg1404647

cg24065044

cg23165623

cg14076258

cg11839863

cg21282997

cg10395772

cg26822501

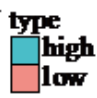

Figure 9

The verification of the stability and reliability of the prognosis prediction model for patients with hepatocellular carcinoma in the training sets. (A) The prognostic differences between high- and low-risk groups in the training set. (B) The distribution of risk scores in high- and low-risk groups in the training 
set. (C) The relationship between risk score and survival status in the training set (as the risk score increases, the number of deaths gradually increases). (D) The distribution of methylation sites between high- and low-risk groups in the prognostic model (from the low-risk group to the high-risk group, the methylation level of each methylation site gradually decreases). (E) The receiver operating characteristic (ROC) curve in the training set. AUC: area under the curve.

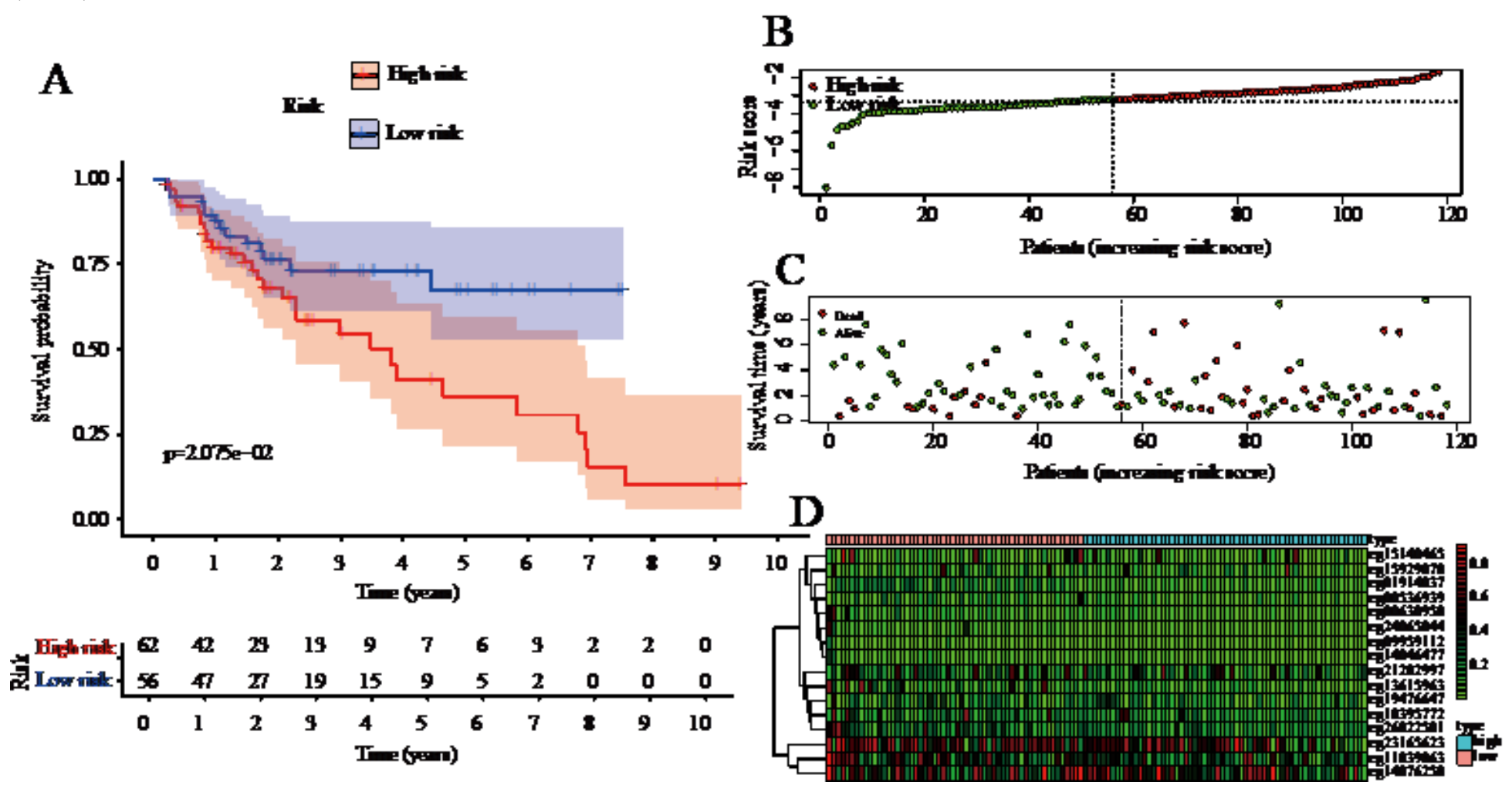

Figure 10

The verification of the stability and reliability of the prognosis prediction model for patients with hepatocellular carcinoma in the test set. (A) The prognostic differences between high- and low-risk groups in the test set. (B) The distribution of risk scores in high- and low-risk groups in the test set. (C) The relationship between risk score and survival status in the test set (as the risk score increases, the number of deaths gradually increases). (D) The distribution of methylation sites between high- and lowrisk groups in the prognostic model (from the low-risk group to the high-risk group, the methylation level of each methylation site gradually decreases). 


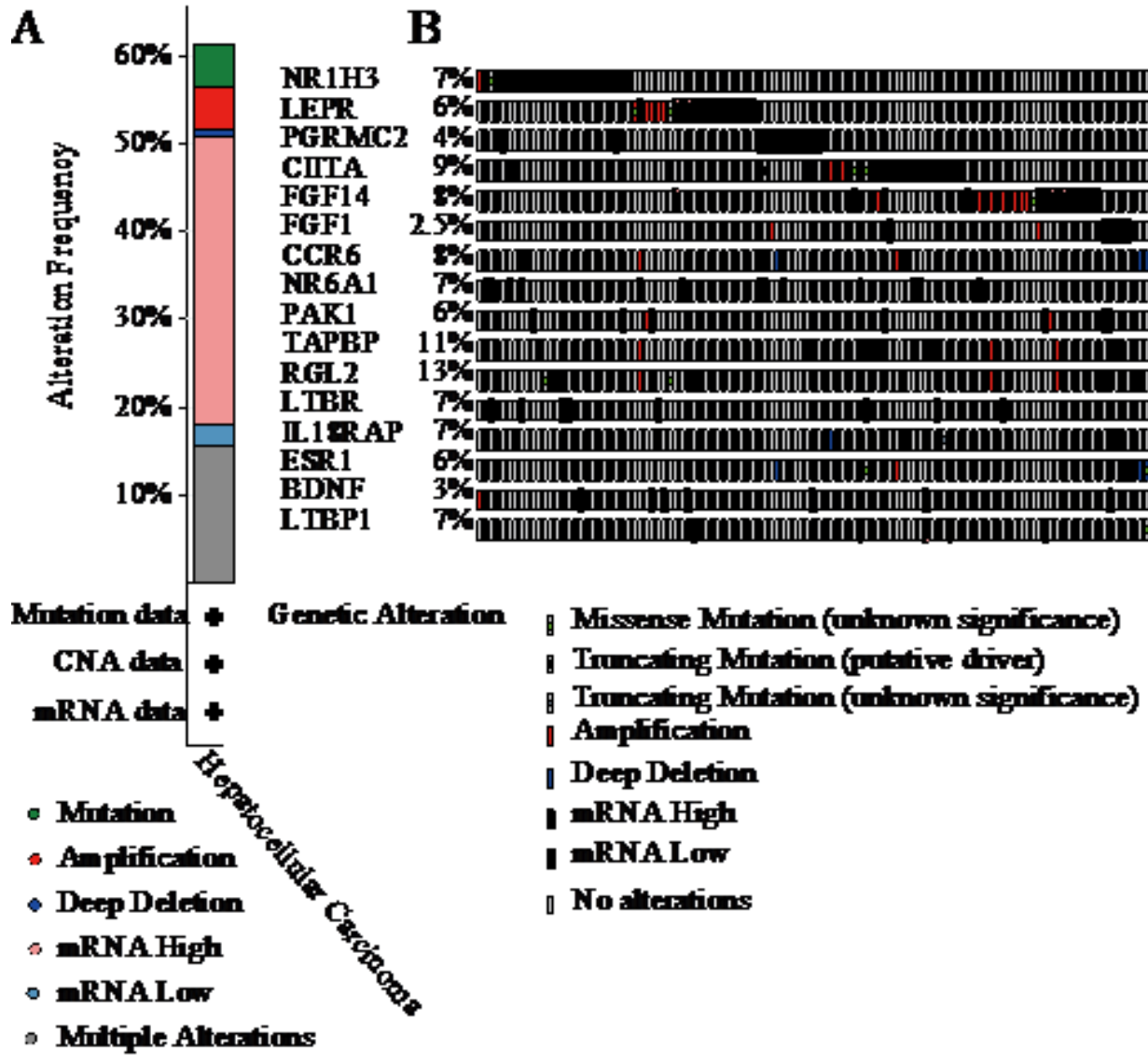

Figure 11

Genetic alterations of immune-related genes driven by $16 \mathrm{CpG}$ sites in the model. 

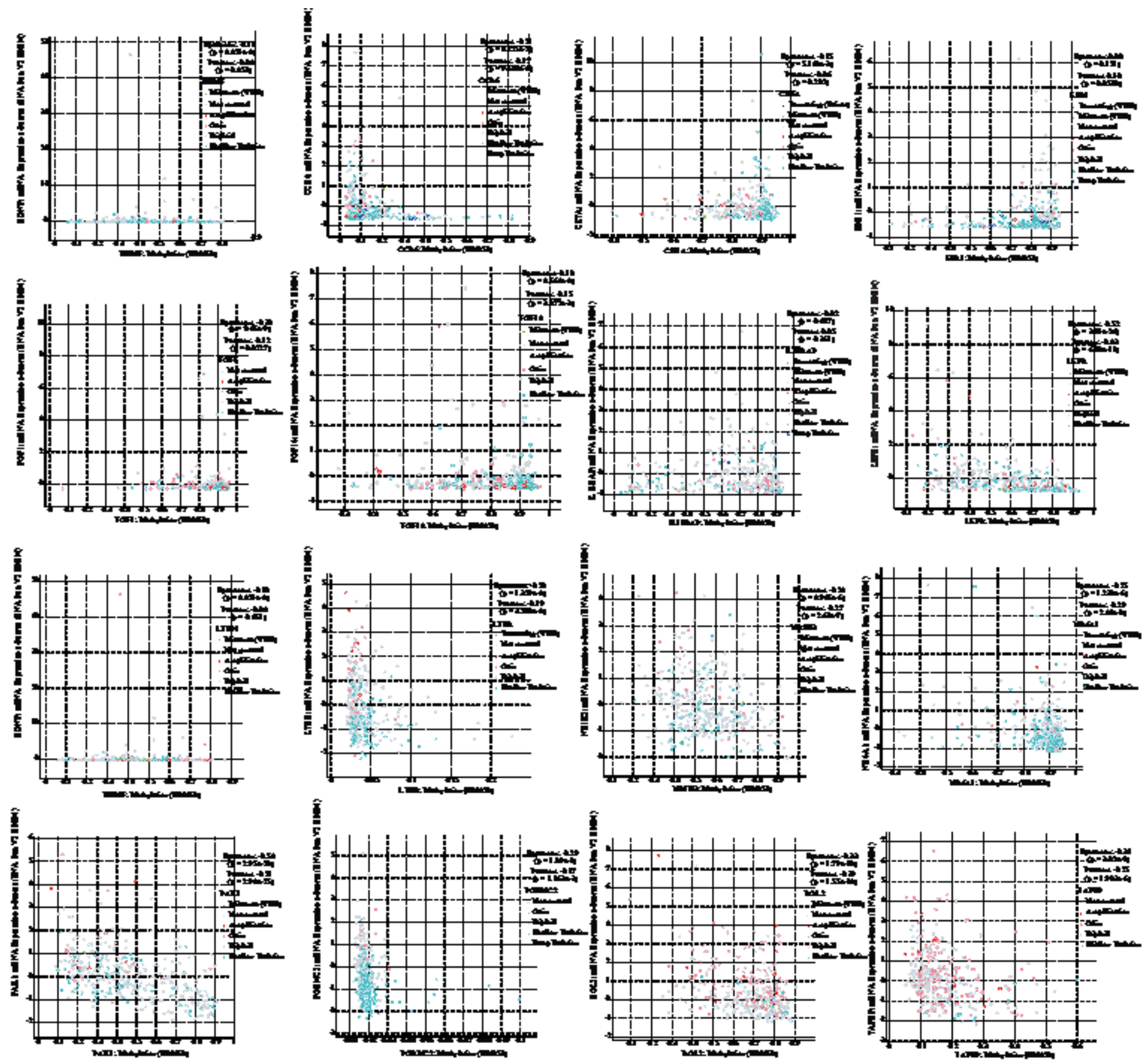

Figure 12

Pearson analysis between the DNA methylation levels of $16 \mathrm{CpG}$ sites in the model and the expression of immune-related genes driven by DNA methylation sites. 

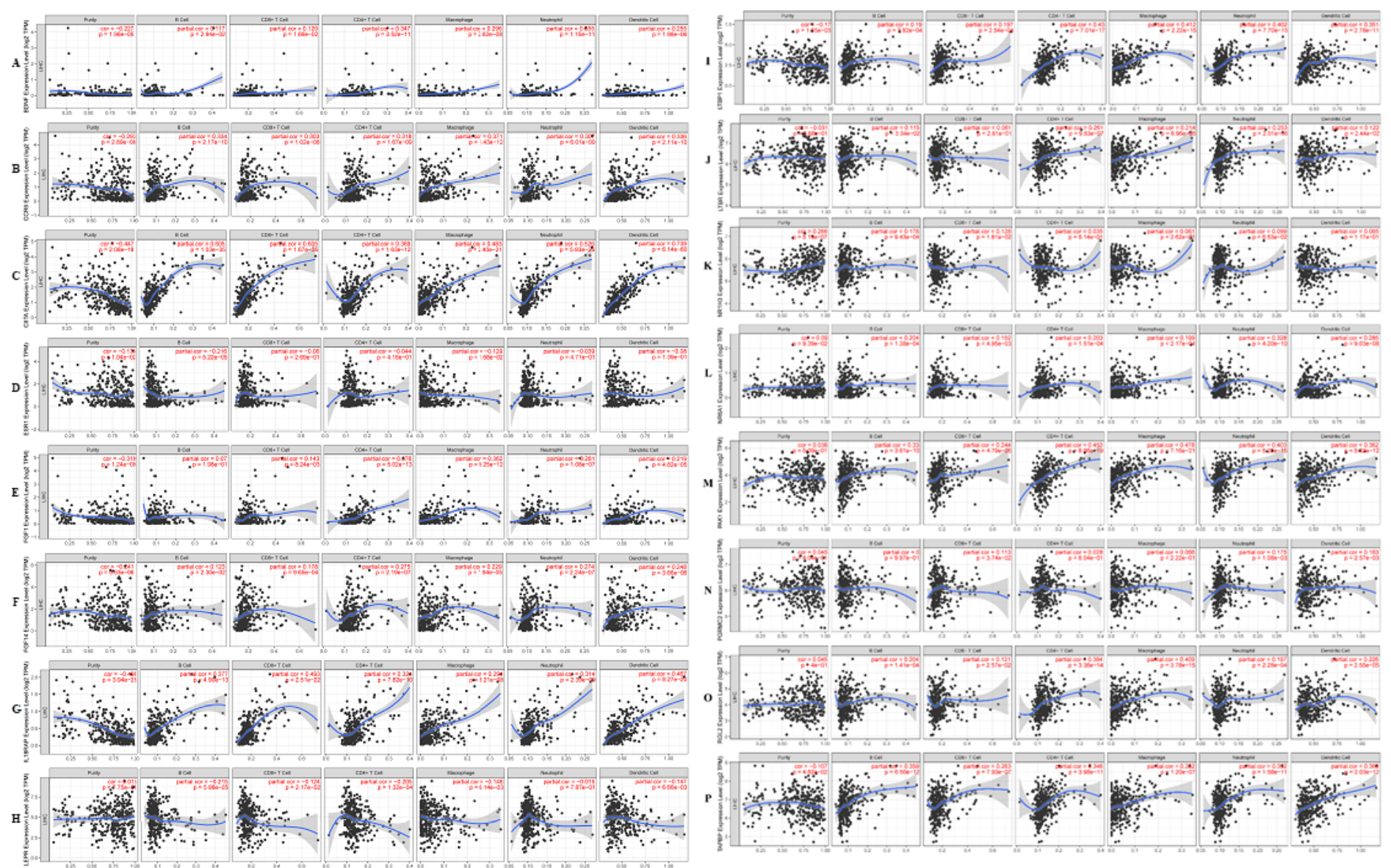

\section{Figure 13}

Correlation of 16 immune-related genes expression with immune infiltration level in LIHC. (A) BDNF expression has significant negative correlation with tumor purity and significant positive correlation with infiltrating levels of B cell, CD8+ T cell, CD4+ T cell, macrophage, neutrophil and dendritic cell.(B) CCR6 expression has significant negative correlation with tumor purity and significant positive correlation with infiltrating levels of B cell, CD8+ T cell, CD4+ T cell, macrophage, neutrophil and dendritic cell.(C) CIITA expression has significant negative correlation with tumor purity and significant positive correlation with infiltrating levels of B cell, CD8+ T cell, CD4+ T cell, macrophage, neutrophil and dendritic cell.(D) ESR1 expression has significant negative correlation with tumor purity, infiltrating levels of $B$ cell and macrophage and no relation with infiltrating levels of CD8+ T cell, CD4+ T cell, neutrophil and dendritic cell.(E) FGF1 expression has significant negative correlation with tumor purity, significant positive correlation with infiltrating levels of CD8+ T cell, CD4+ T cell, macrophage, neutrophil and dendritic cell and no relation with infiltrating levels of B cell.(F) FGF14 expression has significant negative correlation with tumor purity and significant positive correlation with infiltrating levels of $B$ cell, CD8+ T cell, CD 4+ $T$ cell, macrophage, neutrophil and dendritic cell.(G) 1L18RAP expression has significant negative correlation with tumor purity and significant positive correlation with infiltrating levels of B cell, CD8+ T cell, CD4+ T cell, macrophage, neutrophil and dendritic cell.(H) LEPR expression has no relation with tumor purity and infiltrating levels of neutrophil and significant negative correlation with infiltrating levels 
of B cell, CD8+ T cell, CD4+ T cell, macrophage and dendritic cell.(I) LTBP1 expression has significant negative correlation with tumor purity and significant positive correlation with infiltrating levels of B cell, CD8+ T cell, CD4+ T cell, macrophage, neutrophil and dendritic cell.(J) LTBR expression has no relation with tumor purity and infiltrating levels of $\mathrm{CD} 8+T$ cell and significant positive correlation with infiltrating levels of B cell, CD4+ T cell, macrophage, neutrophil and dendritic cell.(K) NR1H3 expression has significant positive correlation with tumor purity and infiltrating levels of B cell, CD8+ T cell, neutrophil and no relation with infiltrating levels of $C D 4+T$ cell, macrophage and dendritic cell.(L) NR6A1 expression has significant positive correlation with tumor purity and infiltrating levels of $B$ cell, CD8+ T cell, CD4+ $T$ cell, macrophage, neutrophil and dendritic cell.(M) PKA1 expression has no relation with tumor purity and significant positive correlation with infiltrating levels of B cell, CD8+ T cell, CD4+ T cell, macrophage, neutrophil and dendritic cell.(N) PGRMC2 expression has no relation with tumor purity and infiltrating levels of $B$ cell, $C D 4+T$ cell, macrophage and significant positive correlation with infiltrating levels of CD8+ T cell, neutrophil and dendritic cell.(0) RGL2 expression has no relation with tumor purity and significant positive correlation with infiltrating levels of $B$ cell, CD8+ T cell, CD4+ T cell, macrophage, neutrophil and dendritic cell.(P) TAPBP expression has significant negative correlation with tumor purity and significant positive correlation with infiltrating levels of B cell, CD8+ T cell, CD4+ T cell, macrophage, neutrophil and dendritic cell. LIHC, liver hepatocellular carcinoma. $p<0.05$ is considered as significant. 